\title{
Identificación de las capacidades tecnológicas y de investigación de la Escuela de Suboficiales y Nivel Ejecutivo Gonzalo Jiménez de Quesada
}

DOI: https://doi.org/10.21158/01208160.n86.2019.2302

Fecha de recepción: 13 de marzo de 2018

Fecha de aprobación: 28 de marzo de 2019
León Darío Parra-Bernal ${ }^{1}$

Universidad EAN

1dparra@universidadean.edu.co

Milenka Linneth Argote-Cusi ${ }^{2}$

Universidad EAN

mlargote@universidadean.edu.co

Lucy Alcira Montoya-Parraga ${ }^{3}$ Escuela de Suboficiales Gonzalo Jiménez de Quesada

lucymonpa@hotmail.com

Nelly Martinez-Rozo ${ }^{4}$

Escuela de Suboficiales Gonzalo Jiménez de Quesada nelly.martinez1165@gmail.com

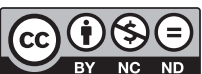

Cómo citar este artículo / To reference this article / Comment citer cet article / Para citar este artigo:

Parra-Bernal, L. D.; Argote-Cusi, M. L.; Montoya-Parraga, L. A.; Martinez-Rozo. N. (2019). Identificación de las capacidades tecnológicas y de investigación de la Escuela de Suboficiales y Nivel Ejecutivo Gonzalo Jiménez de Quesada. Revista Escuela de Administración de Negocios, (86), 207-225. DOI: https://doi.org/10.21158/01208160.n86.2019.2302

\section{Resumen}

En el plan nacional de desarrollo «prosperidad para todos» en sus capítulos VII y VIII se resaltó la importancia de fortalecer el sistema nacional de ciencia y tecnología, a partir de la articulación entre la Universidad, el Estado y el sector productivo. Para que dicha articulación surta efecto, se hace necesario el fortalecimiento de capacidades institucionales de transferencia de resultados de investigación, que sirvan como puentes facilitadores entre las necesidades de la sociedad y el sector privado y público, y la oferta de productos en ciencia y tecnología emanada de cada universidad, que pueda dar solución total o parcial a dichas necesidades.

Pensando en ello, el proyecto del cual surge este artículo tuvo como propósito caracterizar el estado actual del proceso de transferencia de resultados de investigación y gestión del conocimiento, que ha realizado la escuela de suboficiales de la policía, Gonzalo Jiménez de Quesada, como un caso de estudio que documenta la metodología utilizada para mapear las capacidades de transferencia y gestión del conocimiento en una organización. De

${ }^{1}$ Economista con especialización en Gestión de Proyectos y ONG de la Universidad Complutense de Madrid. Maestro en Población y Desarrollo por la Facultad Latinoamericana de Ciencias Sociales - FLACSO, sede México -. Estudios de doctorado en economía por la Universidad Nacional Autónoma de México (UNAM). ORCID: https://orcid.org/0000-0002-1509-1262

${ }^{2}$ Magister en Demografía Estadística por la Facultad Latinoamericana de Ciencias Sociales sede México. Ingeniera de Sistemas por la Escuela Militar de Ingeniería de Bolivia. ORCID: https://orcid.org/0000-0003-4448-1347

${ }^{3}$ Licenciada en español y Literatura de la Universidad del Tolima. Especialista en Administración de la Informática Educativa de la Universidad de Santander UDES. Especialista en la Enseñanza de las Ciencias Sociales e Investigación por la Universidad Internacional FLACSO de Argentina. Magister en Gestión de la Tecnología Educativa por la Universidad de Santander UDES. ORCID: https://orcid.org/0000-0002-7168-9111

${ }^{4}$ Licenciada en administración educativa de la Universidad San Buenaventura. Especialista en investigación de accidentes de tránsito por la Escuela de seguridad vial Policía Nacional General Deogracias Fonseca. Magister en dirección y gestión de instituciones educativas. Doctoranda en Educación Universidad Unini de México. ORCID: https://orcid.org/0000-0001-5534-2807 
acuerdo con lo anterior, uno de los principales hallazgos de la investigación fue identificar cuáles serían los principales cuellos de botella para mejorar el proceso de desarrollo y maduración de los proyectos y productos de investigación, susceptibles de ser transferidos al sector público. Se observó que el mayor obstáculo a superar es la continuidad de los proyectos o iniciativas, bajo nivel de divulgación de las mismas, falta de capacitación y el desarrollo de tutoriales para la implementación de estos.

Palabras clave: investigación; capacidades tecnológicas; gestión del conocimiento; transferencia de conocimiento; capacidades de investigación; capacidades de transferencia; sistema nacional de ciencia y tecnología.

\title{
Identification of the technological and research capacities in the School for Non-Commissioned Officers and Executive Level Gonzalo Jimenez de Quesada
}

\begin{abstract}
The National Development Plan "Prosperidad para Todos", in its chapters VII and VIII highlighted the importance of strengthening the national system of science and technology based on the articulation among the University, the Government, and the Production Industry. For this articulation to take effect, it is necessary to strengthen the capacities of the institutions to transfer research results, which work as facilitating bridges between the needs of society and the private and public sectors, and the supply of science and technology products generated in each university, which can give total or partial solution to those needs.

Based on that, the project from which this article arises had the purpose of characterizing the state of the art of the current process of transferring research and knowledge management results that has been carried out by the School for Non-commissioned Police Officers,Gonzalo Jimenez de Quesada, as the case study that documents the methodology that was used to map their capabilities for knowledge management and transfer in an organization. According to the above, one of the main findings of the research was to identify what the main obstacles to improve the processes of development and maturation of research projects and products subjected to be transferred to the public sector would be. It was observed that the biggest obstacle to overcome is the continuity of the projects or initiatives, the low level of disclosure, and the lack of training and development of tutorials for their implementation.
\end{abstract}

Keywords: research, technological capacities, knowledge management, knowledge transfer, research capacities, transfer capacities, National Science and Technology System.

\section{Identificação das capacidades tecnológicas e de pesquisa da Escola de Suboficiais e Nível Executivo Gonzalo Jiménez de Quesada}

\section{Resumo}

No plano de desenvolvimento nacional "prosperidade para todos" nos Capítulos VII e VIII se ressaltou a importância do fortalecimento do sistema nacional da ciência e tecnologia a partir da articulação entre a Universidade, o Estado e o setor produtivo. Para que tal articulação produza efeitos, se faz necessário o fortalecimento da capacidade institucional de transferência dos resultados de pesquisa que servem como pontes facilitadores entre as necessidades da sociedade e os setores público e privado e ofertas de produtos em ciência e 
tecnologia emanada de cada universidade, que pode dar solução total ou parcial a essas necessidades.

Pensando nisso, o projeto do qual surge este artigo, teve como objetivo caracterizar o estado atual do processo de transferência de resultados de investigação e gestão do conhecimento, realizado pela escola de suboficiais da polícia, Gonzalo Jiménez de Quesada, como um caso de estudo que documenta a metodologia usada para mapear as capacidades de transferência e gestão do conhecimento em uma organização. De acordo com o exposto, um dos principais resultados da pesquisa foi identificar quais seriam os principais pontos de estrangulamento para melhorar o processo de desenvolvimento e maturação de projetos e produtos de pesquisa, que possam ser transferidos para o setor público.

Notou-se que o maior obstáculo a superar é a continuidade dos projetos e iniciativas, o baixo nível de divulgação dos mesmos, e a falta de formação e desenvolvimento de tutoriais para implementar estes.

Palavras-chave: pesquisa; capacidades tecnológicas; gestão do conhecimento; transferência de conhecimento; capacidades de pesquisa; capacidades de transferência; sistema nacional de ciência e tecnologia.

\section{Identification des capacités technologiques et d'investigation de l'École d'Officiers et Sous-Officiers Gonzalo Jiménez de Quesada}

\section{Résumé}

Les chapitres VII et VIII du plan national de développement baptisé “Prospérité pour tous" souligne l'importance du renforcement du système national pour les sciences et la technologie basé sur une meilleure coordination entre les universités, l'État et le secteur productif. Lamélioration de cette coordination passe par un renforcement des capacités institutionnelles en matière de transfert des résultats d'investigations académiques servant de passerelles entre les besoins de la société et des secteurs privé et public avec l'offre de produits scientifiques et technologiques émanant de chaque université et pouvant résoudre partiellement ou totalement ces besoins.

Le projet à l'origine de cet article avait pour but de caractériser l'état actuel du processus de transfert des résultats d'investigations et de gestion des connaissances réalisé par l'école des sous-officiers de police Gonzalo Jiménez de Quesada. Cette étude documente la méthodologie utilisée pour cartographier les capacités de transfert et de gestion des connaissances dans une organisation. L'un des résultats de cette investigation identifie les principaux obstacles à l'amélioration du processus de développement et de maturité des projets pouvant être transférés au secteur public. Les principaux obstacles à surmonter ont trait à la continuité des projets ou des initiatives, à leur faible niveau de divulgation, au manque de formation et au développement de tutoriels pour leur mise en œuvre.

Mots-clés: recherche; capacités technologiques; gestion des connaissances; transfert de connaissances; capacités de recherche; capacités de transfert; système national des sciences et des technologies. 


\section{Introducción}

$\mathrm{U}$ no de los caminos hacia el desarrollo económico es el fortalecimiento del Sistema Nacional de Innovación (SNI). Diferentes estudios han demostrado que el nivel de desarrollo tecnológico, la inversión en $\mathrm{I}+\mathrm{D}+\mathrm{I}$ y el capital humano potencian el nivel de productividad de una nación y así su desarrollo. No es difícil deducir que los factores mencionados están relacionados con el sistema educativo, ya que desde allí es que se pueden generar las innovaciones (aunque no son los únicos, puesto que las empresas también innovan) y se forma capital humano, detonantes clave para el desarrollo (Cimoli, 2000; Parra-Bernal y Argote-Cusi, 2017, 2018a y 2018b; Porter y Stern, 2001; Turriago-Hoyos, 2014).

La transferencia de estas innovaciones y conocimiento de las instituciones educativas hacia el sector externo se conoce como transferencia tecnológica. Es necesario aclarar que esta función de transferencia se adjudica sobre todo a las universidades debido a que ellas son las que realizan de manera formal y cuentan con los recursos para investigación; sin embargo, considerando el contexto colombiano en que el nivel técnico tiene un peso importante, es factible involucrar estas instituciones educativas en este proceso, entre ellas las instituciones formadoras de otros sectores como el de la Policía Nacional de Colombia, que cuenta con diferentes escuelas de formación unidas a su práctica diaria.

Los procesos de transferencia tecnológica son complejos y América Latina se encuentra en una etapa incipiente en esta área. Países como Chile, México y Argentina cuentan en su sistema con oficinas de transferencia tecnológica (OTT); sin embargo, el país más experimentado en este sentido son los Estados Unidos que desde la década de 1980 con la Ley BayhDole, fomenta la creación de patentes y la normativa para el desarrollo de esta (Rothaermel, Agung y Jiang,
2007). De acuerdo con Ranga, Temel, Ar, Yesilay y Sukan (2016), uno de los aspectos más conflictivos para el desarrollo de los procesos de transferencia son los derechos de propiedad intelectual de las universidades y la idiosincrasia de las naciones para emprender estos procesos (Argote-Cusi, 2016, 2017). Las variables culturales y sociales de los diferentes espacios geográficos definen una dinámica en la que puede ser positiva o negativa para el desarrollo de la transferencia tecnológica exitosa (Katz, 1986, 2000, 2007).

Un paso previo a la transferencia tecnológica es la identificación de las capacidades tecnológicas para determinar lo que se puede transferir. Como veremos en las siguientes secciones, la investigación sobre capacidades tecnológicas en el sector industrial es muy amplia; sin embargo, algunos trabajos como Argote-Cusi $(2016,2017)$ extrapolan este conocimiento al sector educativo con su investigación sobre las capacidades tecnológicas de una universidad especializada en el área de la salud en Colombia.

La universidad, vista como una organización, es susceptible de ser analizada desde el punto de vista de sus capacidades e identificar aquellas actividades que generan innovación y aprendizaje con el objetivo de medir estas capacidades para la toma de decisiones de transferencia tecnológica. Esta investigación retoma el modelo conceptual, la metodología y los instrumentos aplicados en la identificación de las capacidades tecnológicas para aplicar en la identificación de las capacidades tecnológicas de la Escuela de Suboficiales y Nivel Ejecutivo Gonzalo Jiménez de Quezada (Esjim) de la Policía Nacional de Colombia dedicada a la formación integral de suboficiales y mandos del nivel ejecutivo a fin de satisfacer las necesidades de la comunidad sobre convivencia y seguridad ciudadana. 


\section{2. ¿Qué es capacidad tecnológica?}

$\mathrm{E}$ sta investigación sobre la identificación de las capacidades tecnológicas de la Esjim requiere como base conceptual la definición de lo que significa capacidad y capacidad tecnológica. El concepto de capacidad en el ámbito organizacional proviene de la teoría de los recursos cuya principal hipótesis es que contar con ciertas capacidades permite a las organizaciones e instituciones desarrollarse de forma exitosa en el tiempo a través de un continuo proceso de retroalimentación, aprendizaje e innovación (Argote-Cusi, 2016, 2017; Domínguez y Brown, 2004; Parra-Bernal y Argote-Cusi, 2017).

Así mismo, el concepto de capacidades tecnológicas describe las habilidades que se caracterizan para iniciar un proceso de mejoras conducentes que permitan un crecimiento y desarrollo sostenido. La definición de capacidades tecnológicas implica conocimientos y habilidades para adquirir, usar, absorber, adaptar, mejorar y generar nuevas tecnologías (Bell y Pavitt,
1995; Lall, 1992). El vacío detectado en relación con el concepto de capacidad tecnológica en la ESJIM es superar la continuidad de los proyectos o iniciativas, bajo nivel de divulgación, además capacitar al personal para fortalecer la tecnología y el desarrollo de tutoriales para su implementación.

Existen diferentes tipos de capacidades analizadas en profundidad en Aguirre-Ramírez (2010), ArgoteCusi $(2016,2017)$ y Domínguez y Brown (2004), entre otros, como se puede ver en forma resumida en la tabla 1. De entre el bagaje de capacidades, las capacidades tecnológicas de innovación se consideran estratégicas en un contexto de grandes y acelerados cambios tecnológicos (Aguirre-Ramírez, 2010). Es en este sentido que la identificación y posterior fortalecimiento de las capacidades tecnológicas de las instituciones toma importancia para ser estudiada en diferentes instituciones privadas y públicas como la Esjim (Katz, 2007).

Tabla 1. Tipos de capacidades por autor

\begin{tabular}{|c|c|}
\hline Referencias & Tipos de capacidades \\
\hline \multirow{5}{*}{ Aguirre (2010) } & Capacidades de investigación y desarrollo \\
\hline & Capacidad de dirección estratégica \\
\hline & Capacidad de mercadeo \\
\hline & Capacidad de fabricación \\
\hline & Capacidad de gestión de recursos \\
\hline \multirow{5}{*}{ Wang et al (2008) } & Capacidades de transferencia \\
\hline & Capacidades de decisión \\
\hline & Capacidades de comercialización \\
\hline & Capacidades de fabricación \\
\hline & Capacidad de capital \\
\hline \multirow{4}{*}{$\begin{array}{c}\text { Brown y Dominguez (2004) } \\
\text { Turriago }(2014)\end{array}$} & Capacidades de producción \\
\hline & Capacidades de inversión \\
\hline & Capacidades de vinculación \\
\hline & Capacidades de innovación \\
\hline \multirow{2}{*}{ Ranga et al (2016) } & Capacidad tecnológica \\
\hline & Capacidad legal \\
\hline \multirow{3}{*}{ Otros } & Capacidad de gestión \\
\hline & Capacidades de aprendizaje \\
\hline & Capacidades de innovación \\
\hline
\end{tabular}

Fuente. Argote-Cusi, 2016, 2017. 
Las capacidades tecnológicas no solo se refieren a la adquisición de nueva tecnológica. En esta investigación, se asume un concepto más amplio que considera los procesos de aprendizaje tecnológico de las organizaciones como procesos clave para la innovación. Para Rousseva (2008), se entiende por capacidad tecnológica la gran variedad de conocimientos y habilidades de las instituciones, necesarios para adquirir, asimilar, utilizar, adaptar, cambiar y crear tecnología. Por otro lado, se entiende que tecnología implica ante todo «transmisión de conocimiento entre distintos agentes» (Domínguez y Brown, 2004, p. 136). Por tanto, las capacidades tecnológicas se refieren a capacidades tangibles como intangibles, y para esta investigación se presta atención a los procesos intangibles en el entendido de que de nada sirve contar con un equipo o maquinaria de última generación si no se utiliza y más aún genera un impacto en la mejora de la organización (en países de economías emergentes donde se adquiere poca tecnología las tecnologías intangibles son una forma práctica de evidenciar la innovación).

Es importante destacar que la investigación de las capacidades tecnológicas se ha dado en torno al sector industrial. Por ejemplo, Domínguez y Brown (2004) desarrollaron un estudio sobre medición de la capacidad tecnológica de las empresas manufactureras mexicanas para lo que sistematizaron las variables a medir, hicieron una selección de las más relevantes para determinar que son las capacidades de producción, las de inversión y vinculación las más estratégicas, así como definen las dimensiones más representativas del aprendizaje tecnológico para medir el aprendizaje tecnológico: la política de formación de personal, la innovación orientada a la mejora continua, los sistemas de información y documentación y la inversión en nuevas tecnologías agrupadas. Por otro lado, Aguirre (2010) llevó a cabo una medición de las capacidades tecnológicas de innovación (CTI) en el sector de desarrollo de software a una muestra de empresas colombianas, de la misma forma que en un trabajo anterior realiza un proceso de sistematización de las variables relacionadas con las capacidades tecnológicas para crear un modelo de lógica difusa.

En esta investigación, se retoma el modelo conceptual de Argote-Cusi $(2016,2017)$ para la identificación de capacidades tecnológicas en instituciones educativas como la Esjim. Se observa a la Esjim desde una perspectiva de análisis organizacional en la que, por ejemplo, las capacidades de producción se refieren a la generación de investigación y de productos innovadores relacionados con la producción de conocimiento; las capacidades de inversión están netamente ligadas a la inversión en $\mathrm{I}+\mathrm{D}+\mathrm{I}$; y las capacidades de vinculación con los grupos de investigación internacional, la relación universidadempresa y la relación universidad-Estado.

Figura 1. Modelo conceptual de las capacidades tecnológicas de la universidad

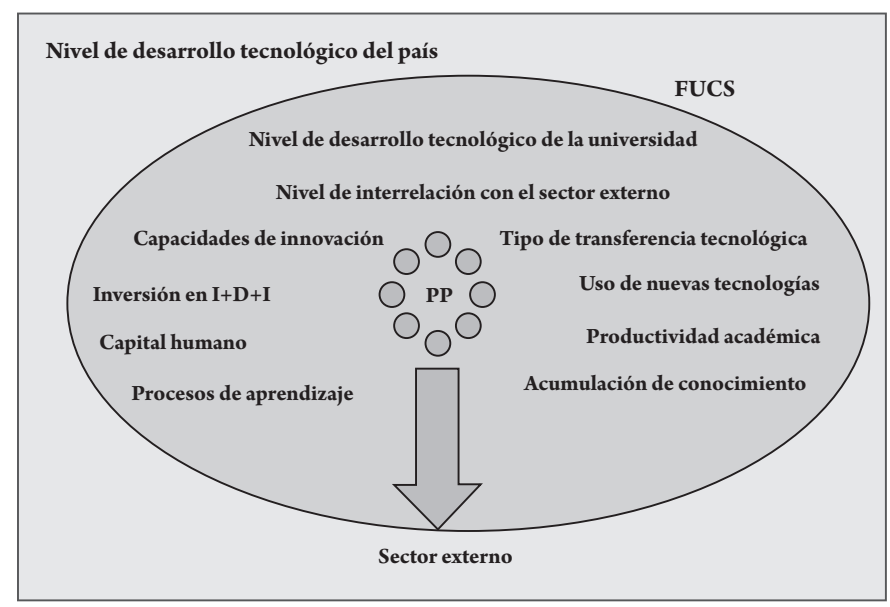

Fuente. Argote-Cusi, 2016, 2017. 
El modelo conceptual de Argote-Cusi (2016, 2017) se materializa en el diseño de instrumentos para la medición de las capacidades tecnológicas de forma cualitativa. Es adecuado optar por una prueba piloto antes de una implementación mayor, para evidenciar la pertinencia de la investigación con los hallazgos. Así es como la investigación de referencia utiliza métodos cualitativos a través de entrevistas semiestructuradas para obtener una muestra de productos con potencial para transferir de la organización estudiada, de acuerdo con la declaración de los actores clave, y para medir de forma cualitativa el ciclo de aprendizaje en relación con nuevas tecnológicas.
El marco teórico permite evidenciar que la identificación de capacidades tecnológicas en las instituciones educativas como las universidades y escuelas de formación policial es una materia nueva en el ámbito del fortalecimiento del SNI y la transferencia tecnológica. Por esta razón, resulta interesante realizar esta investigación aplicable a diferentes organizaciones como la Esjim considerando el modelo conceptual y los hallazgos de Argote-Cusi (2016, 2017), con el objetivo de contar con una línea base acerca del potencial de la organización para la transferencia tecnológica y de las áreas que se requieren fortalecer en $\mathrm{I}+\mathrm{D}+\mathrm{I}$.

\section{Las capacidades de transferencia en las universidades}

Según el Centro Interuniversitario de Desarrollo (Cinda, 2015), se destaca la heterogeneidad del nivel de desarrollo en cuanto a transferencia de $\mathrm{I}+\mathrm{D}+\mathrm{I}$, innovación y emprendimiento en la región. En general. la inversión en $\mathrm{I}+\mathrm{D}+\mathrm{I}$ en la primera década del siglo XXI no ha superado el $2 \%$, de modo que los países que más invierten son España y Portugal. Comparado con los países desarrollados del Sudeste Asiático y de Norteamérica, aún existen brechas en cuanto a financiamiento, sin embargo, se observa un crecimiento de cerca del $7 \%$ en cuanto al número de publicaciones científicas, así como un crecimiento promedio del $3 \%$ anual en el número de patentes generados (2191 patentes en Iberoamérica entre 2003 y 2009).
Respecto de capital humano calificado también se evidencian avances, ya que se registra en promedio un investigador por cada 1000 hab. de la población económicamente activa (PEA), que está en incremento. Es decir, existen esfuerzos de parte de varios países en cuanto a políticas de ciencia y tecnología e inversión en $\mathrm{I}+\mathrm{D}+\mathrm{I}$, cuyos resultados se verán en los próximos años. Los procesos han sido lentos en parte debido al ecosistema productivo de Iberoamérica en torno a la producción y explotación de materias primas que demandan poca tecnología (Cinda, 2015). 


\section{La Escuela de Suboficiales y Nivel Ejecutivo Gonzalo Jiménez de Quesada}

C olombia se encuentra en un momento histórico importante denominado posacuerdo. En la actualidad, la sociedad colombiana tiene gran expectativa acerca de los procedimientos futuros de una institución como es la Policía Nacional, debido a los resultados que se han generado frente al acuerdo de paz logrado con las Fuerzas Armadas Revolucionarias de Colombia (FARC), y los retos que implica el posacuerdo. Firmada la paz con una de las guerrillas más antiguas de América Latina, las instituciones deben afrontar grandes retos en lo que implica el proceso de posconflicto para obtener resultados tangibles a corto, mediano y largo plazo.

En este contexto social, político y económico, la Policía Nacional de Colombia desempeña un papel muy importante al ser «un cuerpo armado permanente de naturaleza civil, a cargo de la nación, cuyo fin primordial es el mantenimiento de las condiciones necesarias para el ejercicio de los derechos y de las libertades públicas, y para asegurar que los habitantes de Colombia convivan en paz» (Constitución Política de 1991, art. 218), por tanto, las escuelas de policía como la Esjim comparten esta misión.

La Esjim, a lo largo de los años de experiencia en la formación de suboficiales y nivel ejecutivo a través de programas académicos dirigidos a la profesionalización y mejora de las competencias de los funcionarios policiales para prestar un servicio de alta calidad a la sociedad, cuenta con diferentes capacidades, sobre todo en formación del talento humano, con el objetivo principal de que la educación policial responda a la Política Nacional de Seguridad y Convivencia Ciudadana.

Como institución educativa, la Esjim cuenta con diferentes programas académicos:

- Tecnología en Estudios Policiales

- Diplomado en Mando Dirección y Liderazgo
- Diplomado en Profesionalización para la Gestión Policial en Seguridad Pública

- Diplomado en Profesionalización para la Gestión Policial en Servicio de Policía y Seguridad Pública

- Diplomado en Profesionalización para la Gestión Policial Convivencia y Seguridad Pública

- Diplomado Profesionalización para la Gestión Policial Análisis y Ejecución del Servicio de Policía

- Curso Nacional e Internacional de Operaciones Especiales

Así también cuenta con un programa de educación a distancia y continúa con una oferta de diferentes cursos en línea.

- Virtual: eventos de capacitación programados por medio de la plataforma del Ministerio de Defensa

- Presencial: desarrollo de eventos académicos por medio de contratos directos con entidades educativas con las que se desarrolló capacitaciones como Diplomado en Doctrina Educativa y Acreditación de Alta Calidad, Diplomado en Funciones Esenciales de la Universidad, Seminario de Gestión del Riesgo, etc.

- Equipos Móviles de Capacitación (Emcap): Seminario de Empleo de Elementos Dispositivos, Municiones y Armas no Letales, Seminario Taller de Actuación Policial, etc.

La Dirección Nacional de Escuelas de la Policía Nacional (Dinae), cuya misión es «direccionar la formación integral del talento humano de la Policía Nacional, a través del sistema educativo policial, en cumplimiento de las funciones de docencia, investigación y proyección social, con el fin de contribuir a la satisfacción de las necesidades de convivencia» (s.f.). cuenta con la Vicerrectoría de Investigación, dependencia encargada de fomentar la cultura investigativa y el desarrollo del sistema de 
ciencia y tecnología de la Policía Nacional. En este marco organizacional, la Esjim tiene la necesidad de fortalecer sus capacidades investigativas y de desarrollo tecnológico considerando que la «la Esjim policial atraviesa cambios vertiginosos, dada la complejidad social actual que demanda un giro en el proceso formativo y de apropiación del conocimiento técnico-científico, en aras de restablecer la credibilidad e importancia institucional como presencia estatal». En este escenario, esta investigación resulta pertinente como primer paso hacia el desarrollo científicotecnológico de la ESJIM. En ese sentido, la pregunta a responder es ¿cuáles son las capacidades tecnológicas de la Esjim que sean factibles de transferir a otras instituciones?

\section{Metodología de mapeo tecnológico}

El tipo de investigación es cualitativa que centra su atención en observar fenómenos y describirlos, para comprender aspectos concretos de la realidad que aún no han sido analizados en profundidad, tales como las dificultades existentes en la transferencia tecnológica y la identificación de capacidades tecnológicas para determinar lo que se puede transferir en la ESJIM. Básicamente, se trata de un primer acercamiento que permite que investigaciones posteriores puedan dirigirse a un análisis profundo de la temática tratada.

Al ser una investigación de tipo descriptivo, el propósito central estuvo orientado a caracterizar el estado actual del proceso de transferencia de resultados de investigación y gestión del conocimiento que ha realizado la ESJIM como un caso de estudio que documenta la metodología utilizada para mapear las capacidades de transferencia y gestión del conocimiento en una organización. Por consiguiente, la importancia de esta investigación radica en el uso de sus resultados como principal insumo para el diseño del plan maestro de gestión del conocimiento de la Esjim.

Así mismo, implementa la metodología de mapeo de capacidades tecnológicas, herramienta que sintetiza de forma gráfica aquellas tecnologías sobre las que se está trabajando en la Esjim, así como su evolución en un determinado periodo.
Según Monzón, Ortega, Martínez y Ortega (2015), «el mapeo tecnológico constituye representaciones visuales del estado de la tecnología en un ámbito o área determinados y se representan gráficamente, de forma sintética, las tecnologías en que se ha investigado más y, en consecuencia, publicado y patentado más en un periodo determinado». De acuerdo con esta definición, la investigación consideró dos frentes de acción: el primer frente se enfocó en el diseño y la implementación de un modelo de transferencia de resultados de investigación que partió del diagnóstico de capacidades institucionales y del diseño de un instrumento de mapeo para identificar, crear y desarrollar productos de investigación susceptibles de ser transferidos al sector público.

La segunda fase estuvo centrada en identificar los productos de investigación y gestión del conocimiento de la Esjim, a partir de la identificación de la fase de desarrollo y maduración de cada producto susceptible de ser transferido al sector público a corto y mediano plazo. En ese sentido, se describió el proceso de maduración de los productos resultado de investigación mediante una adaptación del esquema conceptual elaborado por Yakhlef y Groupe (2007) y López-Torres, Maldonado-Guzmán, Pinzón-Castro y García-Ramírez (2016) de acuerdo con la figura 2. 
Figura 2. Ciclo de maduración y desarrollo producto de investigación transferible



Fuente. Elaboración propia.

Para iniciar la operación del programa de fortalecimiento de capacidades institucionales de transferencia de resultados de investigación de la Esjim, fue necesario construir una línea base que sirva como diagnóstico de capacidades institucionales sobre el estado actual de los productos y resultados de investigación que son susceptibles de ser transferidos al sector público u otras escuelas de policía. Para ello, la primera fase de operación del citado programa se enfocó en diseñar los instrumentos de mapeo de capacidades, a saber, el instrumento de entrevista semiestructurada para cinco expertos e informantes clave de la Esjim encontrados en las áreas académicas de investigación y gestión del conocimiento y las matrices de análisis que sintetizan la información proporcionada por estos (Tabla 2).

Tabla 2. Ciclo del proceso de aprendizaje a partir de la adquisición de nuevas tecnologías tangibles o intangibles

\begin{tabular}{|c|c|c|c|c|c|c|c|c|}
\hline $\begin{array}{c}\text { Nueva tecnología } \\
\text { (tangible o } \\
\text { intangible) }\end{array}$ & $\begin{array}{c}\text { Tipo de } \\
\text { tecnología }\end{array}$ & $\begin{array}{c}\text { Categoría, } \\
\text { Fase de } \\
\text { desarrollo }\end{array}$ & $\begin{array}{c}\text { Se } \\
\text { adquiere }\end{array}$ & $\begin{array}{c}\text { Se } \\
\text { aprende } \\
\text { a usar }\end{array}$ & $\begin{array}{c}\text { Se } \\
\text { asimila }\end{array}$ & $\begin{array}{c}\text { Se } \\
\text { utiliza }\end{array}$ & $\begin{array}{c}\text { Se } \\
\text { mejora }\end{array}$ & Observaciones \\
\hline $\begin{array}{c}\text { Seminario } \\
\text { Internacionalización }\end{array}$ & $\begin{array}{l}\text { Tecnología } \\
\text { blanda }\end{array}$ & $\begin{array}{l}\text { Producto } \\
\text { mínimo } \\
\text { viable }\end{array}$ & $\mathrm{Si}$ & $\mathrm{Si}$ & $\mathrm{Si}$ & $\mathrm{Si}$ & No & $\begin{array}{c}\text { La Escuela hace } \\
\text { parte del proceso de } \\
\text { internacionalización de } \\
\text { la Educación policial, a } \\
\text { través de los convenios } \\
\text { con otros países. }\end{array}$ \\
\hline Plataforma virtual & $\begin{array}{l}\text { Tecnología } \\
\text { blanda }\end{array}$ & Propuesta & $\mathrm{Si}$ & $\mathrm{Si}$ & $\mathrm{Si}$ & $\mathrm{Si}$ & $\mathrm{Si}$ & $\begin{array}{l}\text { Se trata de un canal } \\
\text { de transferencia } \\
\text { importante de } \\
\text { conocimiento entre } \\
\text { la academia y la } \\
\text { función policial, lo que } \\
\text { conduce a actividades } \\
\text { teniendo en cuenta } \\
\text { casos policiales. }\end{array}$ \\
\hline $\begin{array}{l}\text { Plataforma para } \\
\text { consulta en línea }\end{array}$ & $\begin{array}{l}\text { Tecnología } \\
\text { blanda }\end{array}$ & $\begin{array}{l}\text { Producto } \\
\text { terminado }\end{array}$ & $\mathrm{Si}$ & $\mathrm{Si}$ & $\mathrm{Si}$ & $\mathrm{Si}$ & $\mathrm{Si}$ & $\begin{array}{c}\text { Creada para que } \\
\text { docentes y directivos } \\
\text { tengan acceso virtual } \\
\text { a los procesos de } \\
\text { matrícula, campus } \\
\text { virtual, biblioteca, } \\
\text { material de apoyo, } \\
\text { programas educativos. }\end{array}$ \\
\hline
\end{tabular}


Tabla 2. Ciclo del proceso de aprendizaje a partir de la adquisición de nuevas tecnologías tangibles o intangibles

\begin{tabular}{|c|c|c|c|c|c|c|c|c|}
\hline $\begin{array}{l}\text { Página Virtual: } \\
\text { Cuéntele al director } \\
\text { de la ESJIM }\end{array}$ & $\begin{array}{l}\text { Tecnología } \\
\text { blanda }\end{array}$ & Proyecto & $\mathrm{Si}$ & $\mathrm{Si}$ & $\mathrm{Si}$ & No & No & $\begin{array}{l}\text { Página virtual donde } \\
\text { los funcionarios } \\
\text { manifiestan al } \\
\text { director de la } \\
\text { escuela sugerencias, } \\
\text { recomendaciones, } \\
\text { quejas y reclamos. }\end{array}$ \\
\hline $\begin{array}{c}\text { Programa Aulas } \\
\text { Amigas }\end{array}$ & $\begin{array}{l}\text { Tecnología } \\
\text { blanda }\end{array}$ & Prototipo & $\mathrm{Si}$ & $\mathrm{Si}$ & $\mathrm{Si}$ & No & No & $\begin{array}{l}\text { A través de convenios } \\
\text { con otras empresas } \\
\text { tecnologicas se } \\
\text { instalan herramientas } \\
\text { tecnológicas, como } \\
\text { tableros, aulas } \\
\text { inteligentes entre otros. }\end{array}$ \\
\hline $\begin{array}{c}\text { Proyecto Classroom } \\
\text { ESJIM }\end{array}$ & $\begin{array}{l}\text { Tecnología } \\
\text { de punta }\end{array}$ & Proyecto & $\mathrm{Si}$ & $\mathrm{Si}$ & $\mathrm{Si}$ & $\mathrm{Si}$ & No & $\begin{array}{l}\text { Servicio web gratuito } \\
\text { que permite a los } \\
\text { estudiantes policiales y } \\
\text { docentes comunicarse } \\
\text { fácilmente dentro } \\
\text { y fuera del centro } \\
\text { educativo. }\end{array}$ \\
\hline $\begin{array}{c}\text { Sistema de gestión } \\
\text { ambiental }\end{array}$ & $\begin{array}{l}\text { Tecnología } \\
\text { blanda }\end{array}$ & proyecto & $\mathrm{Si}$ & $\mathrm{Si}$ & $\mathrm{Si}$ & $\mathrm{Si}$ & No & $\begin{array}{c}\text { En la Escuela Gonzalo } \\
\text { Jiménez de Quesada } \\
\text {, en el marco de la } \\
\text { responsabilidad } \\
\text { ambiental, se } \\
\text { realizan programas } \\
\text { de protección del } \\
\text { ambiente. }\end{array}$ \\
\hline
\end{tabular}

Fuente. Elaboración propia.

La matriz de la tabla 2 tuvo como propósito identificar tanto las posibles tecnologías desarrolladas por la Esjim como la fase de desarrollo en la que se encontraba el producto para ser transferido. Dicha matriz fue complementada con la tabla 2 en la que se buscó identificar tanto los diferentes actores clave que intervinieron en cada iniciativa como el nivel de articulación interna, internacionalización y cohesión de los investigadores involucrados con el resto de ESJIM, aliados internacionales y su propio grupo de investigación. 


\begin{tabular}{|c|c|c|c|c|c|c|c|c|c|c|}
\hline$\varpi$ & 0 & $\infty$ & $\checkmark$ & $a$ & u & + & $\omega$ & $N$ & - & $\frac{z}{6}$ \\
\hline 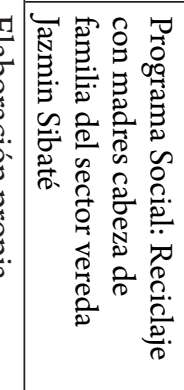 & 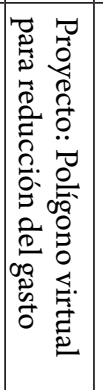 & 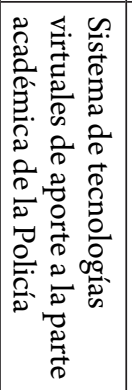 & 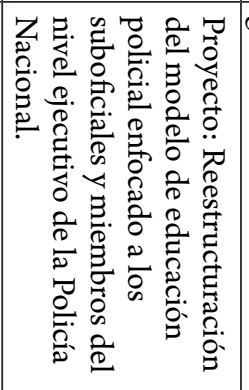 & 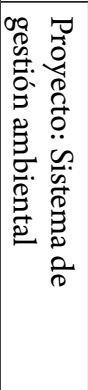 & 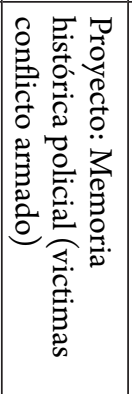 & 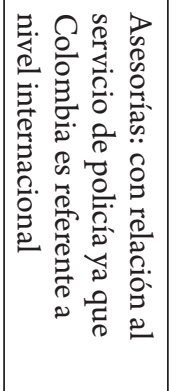 & 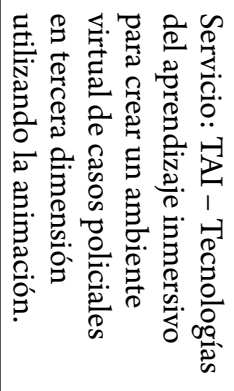 & 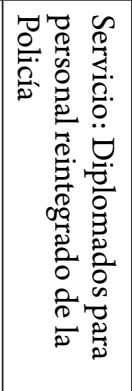 & 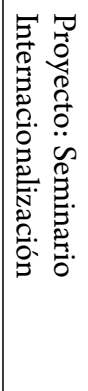 & 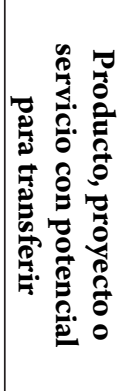 \\
\hline ż & z & ż & $\because$ & z & $\cong$ & z & $\cong$ & $\cong$ & $\because$ & 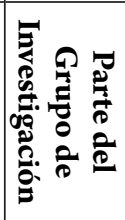 \\
\hline 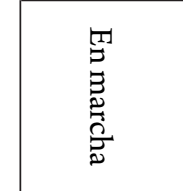 & 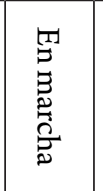 & 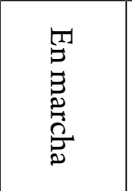 & 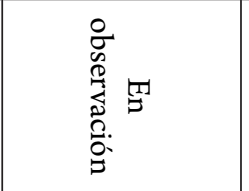 & 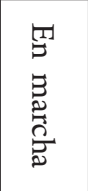 & $\begin{array}{l}5 \\
5 \\
0 \\
0 \\
0 \\
0 \\
0 \\
0\end{array}$ & 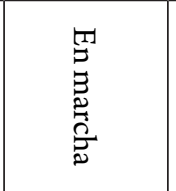 & 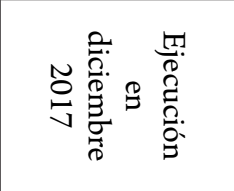 & $\begin{array}{l}\text { TI } \\
\stackrel{5}{0} \\
0 \\
0 \\
0 \\
0 \\
0\end{array}$ & 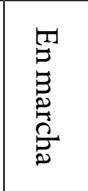 & 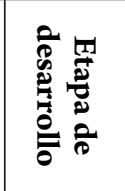 \\
\hline $\begin{array}{l}\frac{3}{0} \\
\frac{0}{0}\end{array}$ & 䗆 & $\underset{0}{\stackrel{D}{0}}$ & $\underset{0}{2}$ & 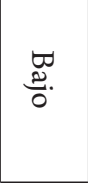 & $\underset{0}{\stackrel{D}{0}}$ & $\underset{0}{\stackrel{D}{0}}$ & $\begin{array}{l}\frac{3}{0} \\
\text { o. } \\
\text {. }\end{array}$ & $\begin{array}{l}3 \\
0 \\
0 \\
0 \\
0\end{array}$ & $\underset{0}{\stackrel{D}{0}}$ & 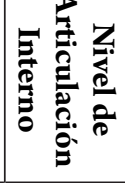 \\
\hline 芯. & 芯. & $\begin{array}{l}\text { Б્ڤ્心 } \\
\text {. }\end{array}$ & $\underset{0}{\stackrel{D}{0}}$ & $\begin{array}{l}\text { t్ట̆ } \\
\text {. }\end{array}$ & $\begin{array}{l}\text { ్ㅠㅇ. } \\
\text {. }\end{array}$ & $\underset{0}{\stackrel{D}{0}}$ & 芯. & & 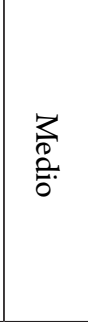 & 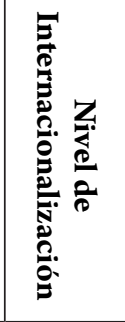 \\
\hline $\begin{array}{l}\frac{3}{0} \\
\frac{0}{0}\end{array}$ & $\underset{0}{\mathbb{2}}$ & $\underset{0}{\stackrel{D}{0}}$ & $\underset{0}{\stackrel{2}{0}}$ & 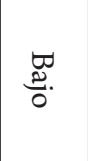 & 芯. & & 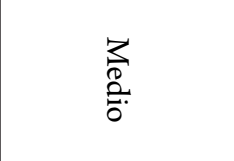 & 芯. & 랑 & 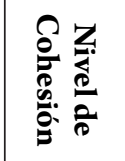 \\
\hline 导 & & $\underset{0}{\vec{D}}$ & $\underset{0}{\vec{D}}$ & 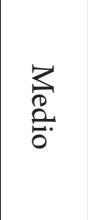 & $\frac{\text { te }}{0}$ & 芯. & 蔍. & 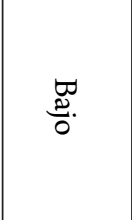 & 胥 & 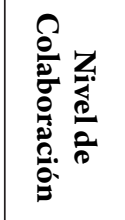 \\
\hline 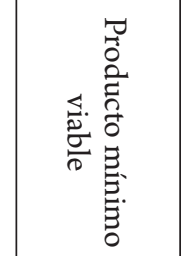 & 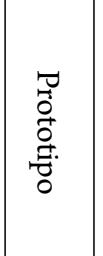 & 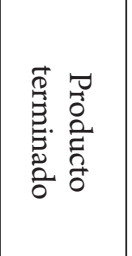 & $\begin{array}{l}\overrightarrow{0} \\
\text { aी } \\
0 \\
\text { ?े }\end{array}$ & 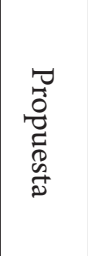 & 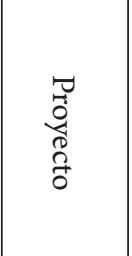 &  & $\underset{D}{\stackrel{2}{D}}$ & 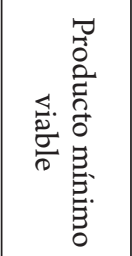 & 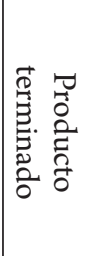 & 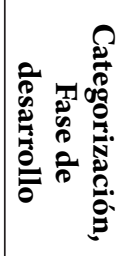 \\
\hline
\end{tabular}




\section{Resultados}

6.1 Desarrollo, uso y adquisición de nuevas tecnologías

De acuerdo con la tipología desarrollada por Thompson en 1967 existen diferentes tipos de clasificación de la tecnología de acuerdo con su grado de obsolescencia, adaptabilidad, flexibilidad y el área en la que se desarrolla. Siguiendo algunas actualizaciones de la anterior tipología, en esta investigación se tomaron tres tipos de tecnología que se adecúan a la naturaleza y objeto social de la Esjim: la tecnología dura que está relacionada con la transformación de materiales, materias primas y objetos tangibles; la tecnología de punta que alude a los últimos avances, mejoras, descubrimientos y desarrollos tecnológicos en cualquier área; y la tecnología blanda asociada al desarrollo de conocimiento intangible que regularmente se plasma en la generación de software, metodologías, nuevas técnicas y modelos de aprendizaje (Shirazi, Mohammadi, Abbasi y Nazari, 2016).

A partir de lo anterior, y considerando lo que respondieron los actores entrevistados, se evidencia en la figura 3 que en la Esjim la tecnología blanda o intangible representa el $71 \%$ de las iniciativas adaptadas o desarrolladas por ella, y deja una baja participación en el uso y desarrollo de tecnologías tangibles, principalmente de punta, con el $29 \%$.

Figura 3. Tipo de tecnología adoptada o desarrollada por la Esjim

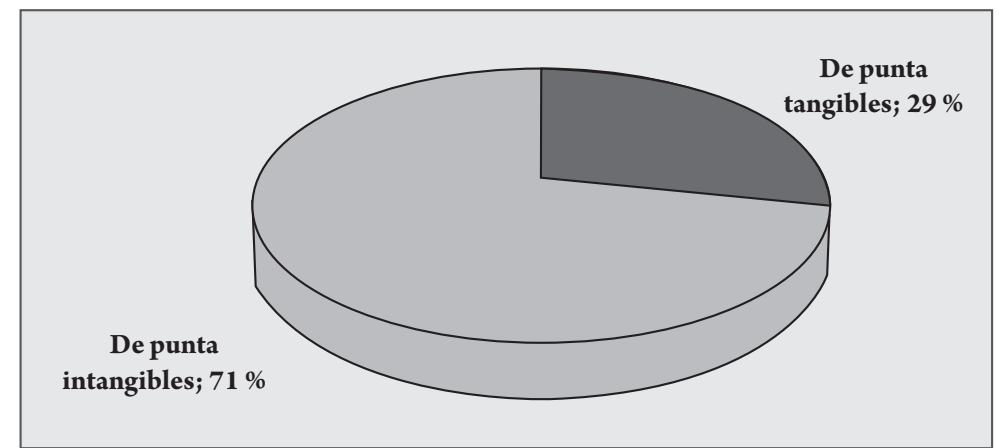

Fuente. Elaboración propia.

Por otro lado, al indagar la adquisición de nuevas tecnologías (inferiores a cinco años en su desarrollo), se encontró que la gran mayoría de las iniciativas implementadas en los últimos años tuvieron este factor presente en los programas académicos y de investigación elaborados por la Esjim. No obstante, al investigar el nivel de asimilación de las tecnologías adquiridas, tres de las siete iniciativas mapeadas no fueron asimiladas completamente por la Esjim como se evidencia en la figura 4. 
Figura 4. Asimilación de la nueva tecnología

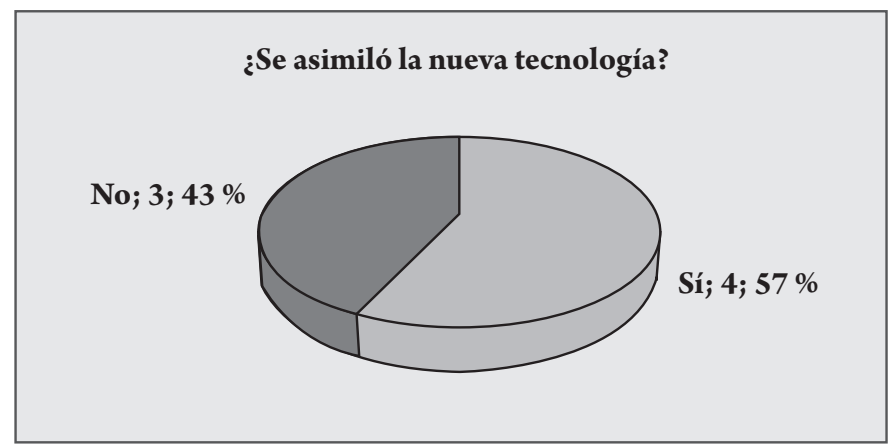

Fuente. Elaboración propia.

Respecto del grado de maduración y desarrollo de productos de investigación conducentes a la implementación de tecnologías susceptibles de ser transferidas por la Esjim al sector público o a otras escuelas de policía, en la figura 5 se observa que un buen porcentaje se encuentra en el nivel de idea $o$ proyecto (terminado o en curso), con un $40 \%$ de las iniciativas; sin embargo, el $60 \%$ restante son productos que están en fase de validación como producto mínimo viable (PMV), prototipo o producto terminado transferible. Ello indica un gran potencial en la Esjim en el desarrollo de productos, principalmente de tecnología blanda, que podrían ser susceptibles de ser transferidos a otras instancias del sector público.

Figura 5. Fase de desarrollo para comercializar

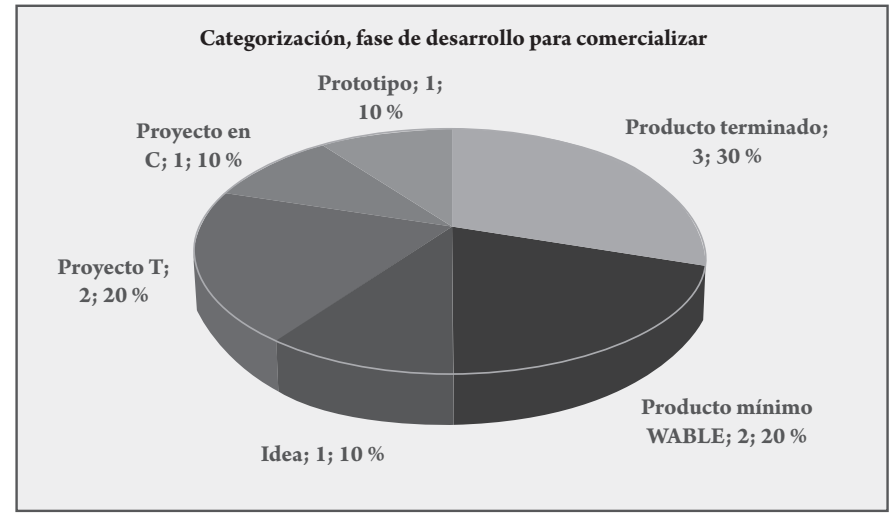

Fuente. Elaboración propia.

\subsection{Articulación de la Escuela de Suboficiales y Nivel Ejecutivo Gonzalo Jiménez de Quesada en investigación}

Una de las dimensiones más importantes para el desarrollo y la maduración de productos y tecnologías susceptibles de ser transferidas en una institución es su nivel de articulación tanto interna como con otras organizaciones nacionales e internacionales. En esta dirección, Sandberg y Crnkovic (2017) indican que la colaboración entre los grupos de investigación, el Estado y el sector privado resulta clave para el desarrollo de tecnología de punta y nuevos avances científicos. Los autores avalan a su vez la necesidad de cooperación entre investigadores de diferentes áreas para el logro y la consecución de impactos sostenibles en los proyectos de investigación. 
Para analizar esta dimensión, se consideraron tres elementos de observación. El primero enfocado en establecer qué porcentaje de las iniciativas que emanan de la Esjim se gesta en su grupo de investigación; en segundo lugar, el nivel de articulación que desde la perspectiva de los actores entrevistados existe en la Esjim; y finalmente el grado de internacionalización de las iniciativas o de los proyectos que se han implementado. En la figura 6, se observa que, de las diez iniciativas, productos o proyectos mapeados, seis fueron gestados en el grupo de investigación EsjimINV, mientras que los otros cuatro fuera de él. Esta situación indica que existe un buen porcentaje de propuestas o productos de investigación que, si bien son susceptibles de ser transferidos, no son articulados a la estrategia de investigación de la Esjim, lo que podría redundar en fallas en el proceso de maduración de las iniciativas y su subsecuente transferencia a otros actores externos a la Esjim.

Figura 6. Proyectos, productos o iniciativas gestadas en el grupo de investigación Esjim-INV

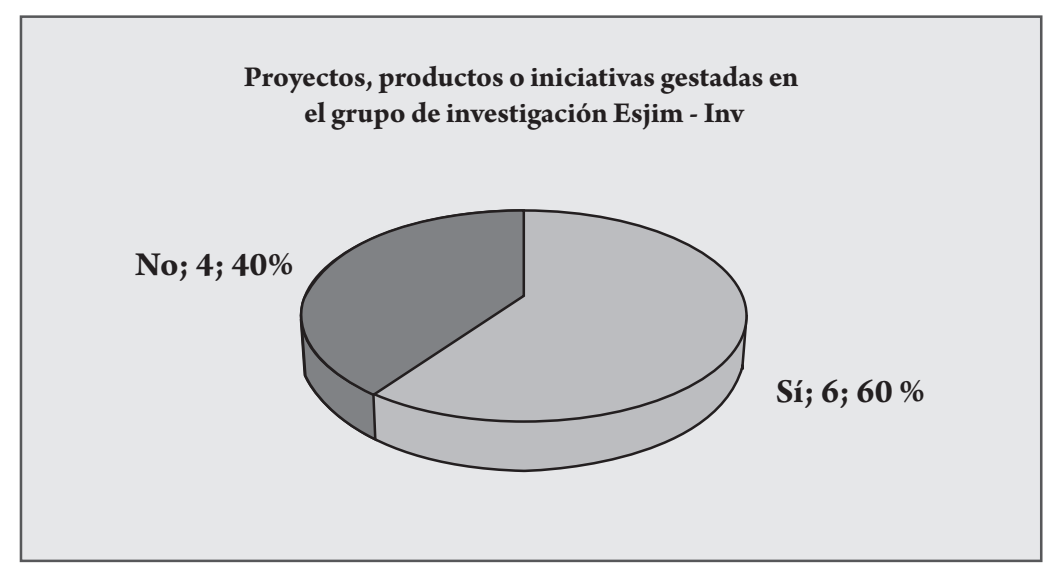

Fuente. Elaboración propia.

En lo referente a la articulación interna, en la figura 7 se observa nuevamente que seis de las diez iniciativas o productos identificados contaban con un alto grado de articulación interna entre las diferentes dependencias de la Esjim para su desarrollo; no obstante, tan solo tres de ellas contaron con un nivel medio o alto de articulación con una entidad internacional para su implementación. Esta situación deja varios aspectos para reflexionar. Primero, si bien el grado de articulación interna es significativo, casi la mitad de los proyectos o productos mapeados se dieron de forma aislada, por iniciativas de carácter individual o emanadas de una sola dependencia; segundo, el bajo nivel de inserción en el campo internacional para el desarrollo de proyectos o productos de investigación da cuenta de la tendencia endógena de la Esjim para la elaboración e implementación de nuevos desarrollos tecnológicos, dado que, a pesar que gran parte de los actores clave entrevistados indicaron que la Esjim tiene una gran proyección hacia el exterior y desarrolla programas de formación y entrenamiento para otros cuerpos de policía en Centroamérica y Suramérica principalmente, en el momento de elaborar y madurar sus productos académicos y de investigación solo lo realiza con su equipo interno. 
Figura 7. Niveles de articulación de Esjim-INV

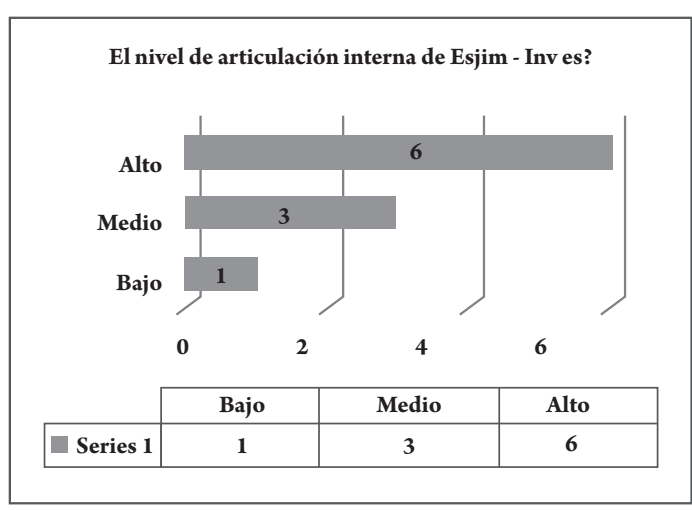

Fuente. Elaboración propia.

Finalmente, se indagó con los actores clave y expertos entrevistados en la Esjim cuáles serían los principales cuellos de botella para mejorar el proceso de desarrollo y maduración de los proyectos y productos de investigación susceptibles de ser transferidos al sector público. En la figura 8, se observa que el mayor



obstáculo a superar es la continuidad de los proyectos o iniciativas con un $50 \%$, seguido del bajo nivel de divulgación con un $29 \%$, la capacitación de los actores involucrados con un $14 \%$ y el desarrollo de tutoriales para la implementación con el $7 \%$.

Figura 8. Principales obstáculos para el desarrollo de iniciativas de nuevo conocimiento

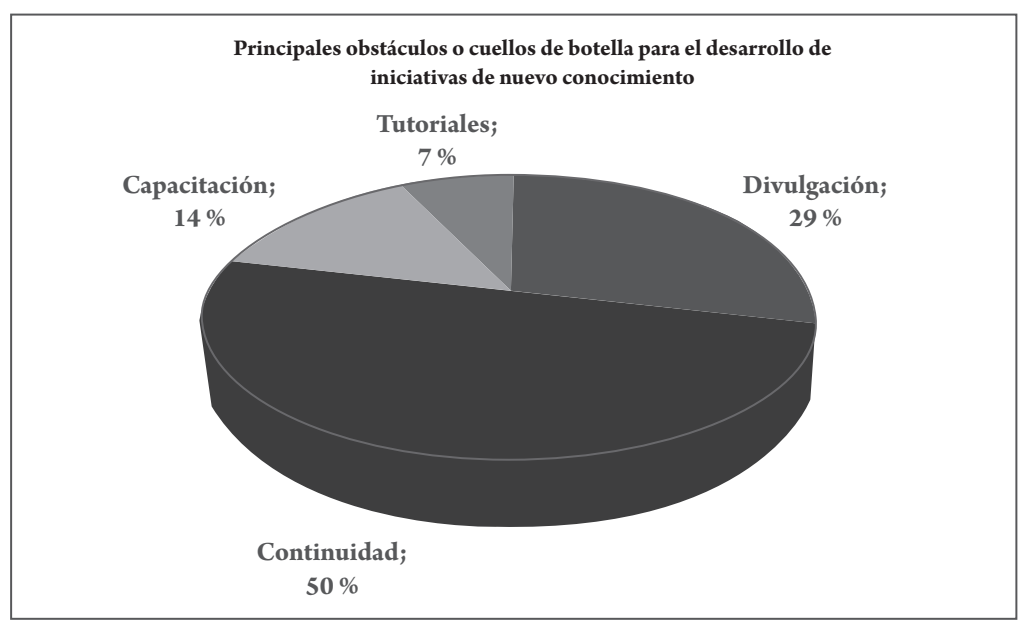

Fuente. Elaboración propia. 


\section{Conclusiones y recomendaciones}

Según el diagnóstico y el análisis obtenido de las entrevistas realizadas a los cinco actores clave de la Esjim, se elaboró el mapeo preliminar de las capacidades de transferencia de resultados de investigación y tecnología sobre el desarrollo y maduración de iniciativas de investigación y productos de tecnología blanda susceptibles de ser transferidos a otras instituciones.

El mapeo permitió identificar los posibles cuellos de botella y obstáculos que los diferentes procesos de investigación y desarrollo han encontrado en el camino para obtener productos terminados con potencial de transferirse al exterior de la Esjim. En este sentido, se generan las siguientes reflexiones finales:

- Se hace necesario focalizar los esfuerzos en desarrollar los productos de gestión de conocimiento y tecnología blanda susceptibles de ser transferidos a otras escuelas de policía tanto a nivel nacional como internacional.

- Garantizar la continuidad de los proyectos o iniciativas de investigación e incrementar los niveles de divulgación.

- Capacitar al personal para fortalecer los procesos investigativos y las tecnologías susceptibles de ser transferidas.

- Desarrollo de tutoriales para implementar la cultura de la innovación en la organización.
También se puede concluir con respecto al proceso de investigación en la Esjim que se deben articular los actores internos con otras instituciones para destacar las capacidades y el desempeño de la Esjim en el fortalecimiento de los procesos formativos al cuerpo policial nacional y el apoyo brindado a diferentes países en tal materia, principalmente en el área de inteligencia, investigación criminal y Plan Nacional de Vigilancia Comunitaria por Cuadrante.

El nivel de articulación interna es alto, dado que todas las áreas de la Esjim están comprometidas con el desarrollo de las iniciativas de investigación. No obstante, a nivel internacional aún existen grandes retos por alcanzar, dado que, pese a la coordinación entre la Oficina de Asuntos Internacionales, la Dirección Nacional de Escuelas y el grupo de investigación Esjim, la mayoría de las iniciativas y de los proyectos se realiza de forma endógena sin la participación de organismos internacionales.

Por último, se concluye que el mayor desafío tanto de la Esjim como de la institución universitaria es darle continuidad a los proyectos y desarrollos de productos de investigación y tecnología que se gestan en la organización, dado que, si bien estos pueden tener un gran potencial de ser transferibles a otras instituciones, no se logra hacerlo en virtud de que los procesos quedan inconclusos o carecen de protocolos y manuales predefinidos para la consecución de productos terminados. 


\section{Referencias}

Aguirre-Ramírez, J. J. (2010). Metodología para medir y evaluar las capacidades tecnológicas de innovación aplicando sistemas de lógica difusa: caso fábricas de software (Tesis de maestría). Universidad Nacional de Colombia, Bogotá, Colombia.

Argote-Cusi,M.L.(2016).Identificación de capacidadestecnológicas de la Fundación Universitaria de Ciencias de la Salud (FUCS). Ponencia presentada en el Primer Congreso Internacional de Innovación y Transferencia de Conocimiento, Quito, Ecuador.

Argote-Cusi, M. L. (2017). Identificación de capacidades tecnológicas de la Fundación Universitaria de Ciencias de la Salud (FUCS). Yura: Relaciones Internacionales, 9.

Argote-Cusi, M. L.; Parra-Bernal, L. D. (2016). Marco conceptual para el análisis de brechas tecnológicas en el sector metalmecánico. En L. D. Parra-Bernal (Coord.), Análisis de brechas tecnológicas en el sector metalmecánico desde el estudio de casos de contraste (pp. 10-34). Bogotá, Colombia: Universidad EAN.

Bell, M.; Pavitt, K. (1995). El desarrollo de capacidades tecnológicas. Comercio, Tecnología y Competitividad Internacional, 22(4831), 69-101.

Centro Interuniversitario de Desarrollo. (2015). La transferencia de $I+D$, la innovación y el emprendimiento en las universidades. Educación superior en Iberoamérica: Informe 2015. Santiago de Chile, Chile.

Cimoli, M. (2000). Creación de redes y sistema de innovación: México en un contexto global. El Mercado de Valores, 60(1), 3-17.

Dirección Nacional de Escuelas de la Policía Nacional. (s. f.). Misión. Recuperado de https://bit.ly/2L4N09M

Domínguez, L.; Brown, F. (2004). Medición de las capacidades tecnológicas en la industria mexicana. Revista de la Cepal, 83. DOI: https://doi.org/10.18356/6cd9babf-es

Escuela de Suboficiales y Nivel Ejecutivo Gonzalo Jiménez de Quesada. (s. f.). Investigación. Recuperado de https://bit. ly/2TYligg

Katz, J. (1986). Desarrollo y crisis de la capacidad tecnológica latinoamericana: el caso de la industria metalmecánica. Buenos Aires, Argentina: Comisión Económica para América Latina y el Caribe. Recuperado de https://bit.ly/2KZvu74

Katz, J. (2000). Reformas estructurales, productividad y conducta tecnológica en América Latina. Santiago de Chile, Chile: Comisión Económica para América Latina y el Caribe. Recuperado de https://bit.ly/2z9CMzB

Katz, J. (2007). Cambios estructurales y ciclos de destrucción y creación de capacidades productivas y tecnológicas en América Latina. Recuperado de https://bit.ly/2PapRrU
Lall, S. (1992). Technological capabilities and industrialization. World development, 20(2), 165-186. DOI: https://doi. org/10.1016/0305-750X(92)90097-F

López-Torres, G. C.; Maldonado-Guzmán, G.; Pinzón-Castro, S. Y.; García-Ramírez, R. (2016). Colaboración y actividades de innovación en Pymes. Contaduría y administración, 61(3), 568-581. DOI: https://doi.org/10.1016/j.cya.2015.05.016

Monzón, M. D.; Ortega, Z.; Martínez, A.; Ortega, F. (2015). Estandarización en la fabricación aditiva: actividades desarrolladas por organizaciones y proyectos internacionales. La revista internacional de tecnología de fabricación avanzada, 76(5-8), 1111-1121.

Parra-Bernal, L. D.; Argote-Cusi, M. L. (2017). Data analytics to characterize university-based companies for decision making in business development programs. En E. Rodriguez (Ed.), Data analytics applications in Latin America and emerging economies (pp. 187-205). Nueva York, EE. UU.: Auerbach Publications. DOI: https://doi.org/10.4324/9781315164113-10

Parra-Bernal, L. D.; Argote-Cusi, M. L. (2018a). Caso de investigación empresarial: Programa de Fortalecimiento de Capacidades en Data Analytics en empresas colombianas. En L. D. Parra Bernal (Coord.), Academia, emprendimiento e investigación empresarial: homenaje a la Universidad EAN en sus 50 años. Bogotá, Colombia: Universidad EAN.

Parra-Bernal, L. D.; Argote-Cusi, M. L. (2018b). Corporate entrepreneurship in Colombia: contrast cases of two colombian manufacturing SMEs. En R. Perez-Uribe, C. Salcedo-Perez y D. Ocampo-Guzman (Eds.), Handbook of research on intrapreneurship and organizational sustainability in SMEs (pp. 368-390). IGI Global. DOI: https://doi. org/10.4018/978-1-5225-3543-0.ch017

Porter, M. E.; Stern, S. (2001). National innovative capacity. En M. E. Porter, J. D. Sachs, P. K. Cornelius, J. W. MacArthur y K. Schwab (Eds.), The global information technology report 20012002 (pp. 102-118). Nueva York, EE. UU.: Oxford University Press.

Ranga, M.; Temel, S.; Ar, I. M.; Yesilay, R. B.; Sukan, F. V. (2016). Building technology transfer capacity in Turkish universities: A critical analysis. European Journal of Education, 51(1), 90106. DOI: https://doi.org/10.1111/ejed.12164

Rousseva, R. (2008). Identifying technological capabilities with different degrees of coherence: The challenge to achieve high technological sophistication in latecomer software companies (based on the Bulgarian case). Technological Forecasting and Social Change, 75(7), 1007-1031. DOI: https://doi. org/10.1016/j.techfore.2007.10.003 
Rothaermel, F. T.; Agung, S. D.; Jiang, L. (2007). University entrepreneurship: A taxonomy of the literature. Industrial and Corporate Change, 16(4), 691-791. DOI: https://doi. org/10.1093/icc/dtm023

Sandberg, A. B.; Crnkovic, I. (2017). Meeting industry: Academia research collaboration challenges with agile methodologies. Ponencia presentada en 39th International Conference on Software Engineering: Software Engineering in Practice Track, Buenos Aires, Argentina. DOI: https://doi.org/10.1109/ ICSE-SEIP.2017.20

Shirazi, A. N. M.; Mohammadi, A.; Abbasi, A.; Nazari, M. (2016). Soft commercial technology and its transfer model in small and medium enterprises: A review. International Journal of Humanities and Cultural Studies, 3(2), 1631-1647. Recuperado de https://bit.ly/2MyhRi7

Thompson, J. (1967). Organizations in action. New York: MacGraw-Hill.

Turriago-Hoyos, Á. (2014). Innovación y cambio tecnológico en la sociedad del conocimiento. (2. ed.). Bogotá, Colombia: ECOE. DOI: https://doi.org/10.2307/j.ctvnltcmq

Yakhlef, A.; Groupe, E. P. (2007). Knowledge transfer as the transformation of context. The Journal of High Technology Management Research, 18(1), 43-57. DOI: https://doi. org/10.1016/j.hitech.2007.03.003 
